\title{
TOPIC 3: IMPACT OF AI AND DEEP LEARNING ON THE HEALTHCARE INDUSTRY
}

\section{INTRODUCTION}

Currently, many healthcare organizations and centers are trying to enhance either managerial or systematic operations. Due to this reason, those organizations have increased their performance and enhanced their financial growth and reputation in local and global markets. Here, deep learning and $\mathrm{AI}$ is used to manage healthcare systems. It helps to provide better service, diagnose multiple diseases and many other things. Based on this fact, the definition of deep learning and $\mathrm{AI}$, the importance and change management using of these tools are going to elaborate in this essay.

The thesis statement aims to focus on deep learning and AI in the healthcare industry, which helps to modify the medical system for patients and other customers.

\section{DEFINE DEEP LEARNING AND A}

Deep learning is a part of machine learning-oriented artificial networks. It has multiple layers that are used to process or extract high-level features from individual data. This deep learning process can enhance data processing level, detect dynamic analysis and many other languages. With the help of this process, computer algorithms can be improved. It can be said that this system can implement in the healthcare industry to enhance the performance of medical systems. This tool helps to modify medical operations, intelligence, and other technical factors. Health workers can diagnose the diseases and analyse them deeply to cure all problems of patients.

Based on this fact, Esteva et al. (2019) stated that deep learning is a machine learning system that increases the computational power to understand multiple data and information. With the help of this tool, data volume is generated and customers can access that data. Apart from this, healthcare organizations can use deep learning to analyse DNA, blood, and other genetic factors. These things are helping to know different data and information; on other hand, all employees or health workers need to gather this learning skill to evaluate medical aspects to negotiate diseases of patients.

In contrast, Miotto et al. (2018) explain deep learning process helps to provide knowledge, heterogeneous and high-dimensional factors. However, electronic health records, biomedical information and other things are effectively managing the complex data challenges. However, it is important to maintain human health for their convenience. Due to this reason, medical operations need to include many advanced technology and data management factors to record all data of patients. Now, it is clear that the deep learning framework is critically enhancing the generalpurpose method of advanced technical aspects. It has several layers to reconstruct errors and connects with each node and system of healthcare systems to get proper outcomes.

Artificial Intelligence (AI) is the most used technology that helps to manage different critical situations. Apart from this, many business domains and organization are focusing on this system to focus on each system that is important to rectify. Panch et al. (2019) stated that AI application is helping to construct sustainable changes in the healthcare industry. All health workers can use this system or tool. Moreover, AI has introduced aggregated healthcare information and data to allow powerful models and maintain dynamic manners. With the help of AI applications, healthcare organizations can diagnose patients and tailor treatments by increasing precision approaches.

By aligning with the previous author, Shaheen, (2021) explain that $\mathrm{AI}$ is a revolutionary technology that enhances strengths, learning factors, and predictable power. In addition, it helps to grasp efficiency to deal with medical systems. AI has increased the ability of health workers, so that they can provide insights into the monitoring method. Medical AI systems have developed their clinical trials to meet all targets. It can also be said that AI applications can improve drug discovery, patient cares, clinical trials and other pharmaceutical firms. These factors have effectively increased the process of healthcare management. Hence, AI is useful to diagnose diseases and improve the quality of life.

Additionally, Pawar et al. (2021) explain regarding AI system. This technology is maintaining all smart systems in healthcare organizations. It helps to enable integrated healthcare technology and application. AI integration can enhance prediction ability, diagnosis ability, personalized connected healthcare and many other things. With the help of this technology, many health workers now understand the significance of AI technology in management domains. This system can diagnose cancer, bone fracture, and other internal defects. All these diseases are very critical; due to this reason, health workers are using this system to be assured regarding this matter.

\section{IMPORTANCE OF AI AND DEEP LEARNING IN THE HEALTHCARE INDUSTRY}

Importance of AI

$\mathrm{AI}$ is a technology that covers individual aspects of healthcare organizations. It creates feasible and reliable facilities to diagnose multiple diseases. On other hand, it can control human health and their well-being. Based on this, AI can be negotiated to increase the healthcare ability of professionals to understand all perspectives in the medical domains. AI provides patients care, feedback, guidance, a healthy lifestyle, and other advice. Due to this reason, health workers are trying to manage their performance to give more importance to AI systems. In this way, the AI system is covering all medical aspects for better improvement.

According to Dave et al. (2020), artificial intelligence helps to modify different systems and rectify functions that bring well-being to the healthcare organization. These authors have stated that the AI system is very important for business management, which can increase financial growth, time management ability; provide skills and knowledge based on some topics. It can be said that AI is helping to manage the healthcare industry, so that organizational members can modify all faults and improve their services to manage medical functions. However, explainable AI can bring potentiality in this domain; manage predictive aspects, programming frameworks, medical diagnosis models, 
trustworthiness and other technical aspects are maintained by this AI system. Therefore, it is clear that without an AI system, healthcare organisations cannot manage their daily performance and they will be unable to enhance their skills and techniques to bring development to healthcare organizations.

In contrast, Mahomed, (2018) opined that AI is associated with high-robotics, which is an automation process. With the help of automation process, the industrial revolution can conduct. Thus, it is clear that AI can manage construction domains, medical, business and other factors. This technology reflects positive influences on health workers. Besides the medical aspects, this system helps to manage legal perspectives, so that no offensive actions can come in the business and medical domains. This is the main importance of the AI system. Hence, AI can bring well-being to healthcare organizations by diagnosing diseases, recording databases, providing healthcare routines and other relevant things.

By aligning with the importance of AI, Davenport, and Kalakota, (2019) stated that many complexities and challenges may come in the healthcare organization. At this moment, health workers need to follow and identify the significance and importance of AI, so that they can modify their activities to achieve success for better development. It can be easy to manage patient engagement, administrative activities, prevent large-scale automation, implement professional jobs and many beneficial aspects are provided by this AI technology. Additionally, it helps to control medical decisions and supports doctors to increase the health quality of patients.

\section{Importance of deep learning}

According to Saba et al. (2019), deep learning is a machine learning system that can increase the efficiency of computer systems. It helps to manage influential aspects in business domains. Here, health workers or doctors need to learn deep learning processes with the help of AI technology, so that they can manage their performance by providing essential treatment to patients. Deep learning is growing in healthcare organizations at a very fast pace. This process can improve quality of life, save decisions; reduce medical costs and radiological benefits. All these areas are needed to develop with the help of deep learning to make a clear decision followed by the compiled deep learning process.

In contrast, Coccia, (2020) explain that deep learning can be applied in emergency cases, such as cancer diagnosis, pathology, technical paradigm, Gartner hype cycle and many other things. These aspects are very important for the healthcare industry. It provides feasibility in cancer imaging at a scientific level. In this case, Amara's law and new technologies are helping to enhance the quality of life, maintain technical management, and do many other things. Deep learning is helping to reduce the current gap, send digital images, and develop regions of diseases. Due to this reason, deep learning is important to enhance healthcare management.

Healthcare industries and organizations are focusing on their performance to provide better healthcare services with the help of machine learning, deep learning, and other technical systems. Based on this matter, Kaur et al. (2018) stated that big data and deep learning is playing a vital role to manage healthcare application. Apart from this, architectural and technical management can be developed by using deep learning. Mask encryption, granular access control, monitoring activity, dynamic data encryption, and other things are incorporated in the deep learning process. Hence, it is clear that deep learning is equally important for the healthcare industry as AI technology.

\section{CHANGE MANAGEMENT IN HEALTHCARE INDUSTRY USING DEEP LEARNING AND AI}

\section{Change management using deep learning}

Deep learning is maintaining the healthcare industry; as a result, this organization can improve their performance and enhance the quality of life. Apart from this, it can say that this learning process brings well-being to healthcare organizations. It changes medical operations; especially, cancer and encryption-oriented factors. These components are creating facilities to change old systems in healthcare management. However, many healthcare organizations are focusing on their responsibilities to implement deep learning systems, machine learning and other relevant processing systems, so that they can enhance their services for better development. Hence, deep learning influence to diagnosis of different diseases to overcome physical and technical aspects.

Based on changing environment of healthcare organizations, deep learning is playing a vital role in medical domains. It helps to make clear insights into disease prediction. According to this point, Sahoo et al. (2019) stated that all employees or health workers needs to participant in technical training sessions to enhance their knowledge of deep learning. This process or tool can change the entire medical process towards success. It reflects a positive impact on patients that is inspiring and motivating for patients. Proper diagnosis processes have changed, healthcare recommendations, clinical pathways, collaborative filtering and other relevant aspects have developed in the healthcare industry. Insurance plans, health environment, medicines recommendations, and many other perspectives can be changed with the help of a deep learning process.

At present, many healthcare organizations are focusing on advanced technology and medical operations, so that proper diagnosis processes can proceed for health development. By aligning with the previous statement, Razzak et al. (2018) explain that healthcare sectors are maintaining their industry and changing medical operational perspectives to enhance their services, where the deep learning process is increasing the knowledge, skills, reducing the percentage of budgets and many other things have changed with the help of deep learning process. Many complexities may come in this sector; at this point, technical development can positively enhance the quality of healthcare services.

\section{Change management using AI}

Artificial intelligence is effectively managing the quality of management and creating beneficial opportunities for the medical industry. With the help of AI, healthcare organizations systems can be changed to get proper services for patients. Apart from this, Reddy et al. (2019) stated to change the technical functions, administration operations, make clinical decisions and many other things. In this way, the healthcare industry can manage their business development and enhance profitability. It can say that public health is more important in the healthcare industry; based on 
this fact, all health workers need to be more attentive and flexible while they are working in the workplace. Here, AI can change the diagnosis systems, prediction developmental process, AI-augmented systems, and many relevant factors. In this way, AI can bring revolution in healthcare organizations.

I contrast, Sun, and Medaglia, (2019) opined that nowadays, AI is maintaining all technical systems and guidelines for individual domains. AI technology is effectively managing the healthcare management, stakeholders, IT systems and other relevant aspects. Here, the AI system conducts diagnostic features for patients. With the help of this technology, all health workers can easily mitigate medical challenges. Due to this reason, AI and deep learning technologies must be implemented to evolve change management in healthcare organizations. AI adoption can change the public sector and represent effective guidelines for beneficial improvement. Besides that, AI is critically managing the decisions, diagnosis factors, administrative perspectives, business development, and many other things.

Regarding AI technology, all intelligence forces can implement new systems in the healthcare organizations, such as remote medical facilities, strategic changes, operational aspects and many other functions. It can say that all health workers can enhance their knowledge, skills, leadership knowledge, medical analysis and other health-oriented factors can get to know with the help of AI technology. Thus, many vague perspectives of healthcare industries can be changed with the help of AI technology. Financial growth, medical knowledge, medicines, patients; information and other healthcare services need to be evolved to bring wellbeing in healthcare organizations. Based on this fact, Garbuio, and Lin, (2019) explain that AI technology or application can bring a revolution in the healthcare industry. All entrepreneurs have to be more conscious and focus on disruptive technologies, innovation, and other healthcare systems. In this case, change management can bring in healthcare organizations.

\section{Methodology}

For this essay, research planning, data analysis, data collection and other procedures are analyses. Here, at first, the idea of the research topic needs to be derived and make decisions to negotiate with this topic. Here, proper knowledge and information are needed regarding AI and deep learning. With the help of internet, journals, and articles, all data are going to collect this process help to provide adequate knowledge of $\mathrm{AI}$ and deep learning. Here, researchers have to focus on healthcare authorities and services. As a result, they will get appropriate information based on importance of deep learning and AI technology.

On other hand, all researchers have to be more focused while analyzing information and data. They have to use effective techniques or any descriptive designs to negotiate the research topic, so that researchers can analyse each section of this essay. With the help of this research, readers and other viewers can get to know the changes management by using deep learning and AI technology. These systems can create revolutionary changes in healthcare domains and other sectors. By following proper research methodology, professional essay study can get to enhance the brief knowledge regarding two advanced technologies.

\section{CONCLUSION}

This essay is based on deep learning and AI technology, which has created many opportunities and facilities in healthcare organizations. In this essay, the descriptive definition of deep learning and AI has been discussed that helps to enhance knowledge regarding this topic. On other hand, the importance of this advanced technology has critically explained that can create new ideas in healthcare organizations. Lastly, change processes by using these technologies can bring well-being in this industry. All health workers and doctors have to gain knowledge regarding these technologies, so that they can provide beneficial services to all patients.

\section{REFERENCES}

[1] Coccia, M., 2020. Deep learning technology for improving cancer care in society: New directions in cancer imaging driven by artificial intelligence. Technology in Society, 60, 101198.

[2] Dave, D., Naik, H., Singhal, S. and Patel, P., 2020. Explainable ai meets healthcare: A study on heart disease dataset. arXiv preprint arXiv:2011.03195.

[3] Davenport, T. and Kalakota, R., 2019. The potential for artificial intelligence in healthcare. Future healthcare journal, 6(2), 94 .

[4] Esteva, A., Robicquet, A., Ramsundar, B., Kuleshov, V., DePristo, M., Chou, K., Cui, C., Corrado, G., Thrun, S. and Dean, J., 2019. A guide to deep learning in healthcare. Nature medicine, 25(1), 24-29.

[5] Garbuio, M. and Lin, N., 2019. Artificial intelligence as a growth engine for health care startups: Emerging business models. California Management Review, 61(2), 59-83.

[6] Kaur, P., Sharma, M. and Mittal, M., 2018. Big data and machine learning based secure healthcare framework. Procedia computer science, 132, 1049-1059.

[7] Mahomed, S., 2018. Healthcare, artificial intelligence and the Fourth Industrial Revolution: Ethical, social and legal considerations. South African Journal of Bioethics and Law, 11(2), 93-95.

[8] Miotto, R., Wang, F., Wang, S., Jiang, X. and Dudley, J.T., 2018. Deep learning for healthcare: review, opportunities and challenges. Briefings in bioinformatics, 19(6), 1236-1246.

[9] Panch, T., Mattie, H. and Celi, L.A., 2019. The "inconvenient truth" about AI in healthcare. NPJ digital medicine, 2(1), 1-3.

[10] Pawar, U., O'Shea, D., Rea, S. and O'Reilly, R., 2020, June. Explainable ai in healthcare. In 2020 International Conference on Cyber Situational Awareness, Data Analytics and Assessment (CyberSA) (1-2). IEEE.

[11] Razzak, M.I., Naz, S. and Zaib, A., 2018. Deep learning for medical image processing: Overview, challenges and the future. Classification in BioApps, 323-350.

[12] Reddy, S., Fox, J. and Purohit, M.P., 2019. Artificial intelligence-enabled healthcare delivery. Journal of the Royal Society of Medicine, 112(1), 22-28.

[13] Saba, L., Biswas, M., Kuppili, V., Godia, E.C., Suri, H.S., Edla, D.R., Omerzu, T., Laird, J.R., Khanna, N.N., Mavrogeni, S. and Protogerou, A., 2019. The present and future of deep learning in radiology. European journal of radiology, 114, 14-24

[14] Sahoo, A.K., Pradhan, C., Barik, R.K. and Dubey, H., 2019. DeepReco: deep learning based health recommender system using collaborative filtering. Computation, 7(2), 25.

[15] Shaheen, M.Y., 2021. Applications of Artificial Intelligence (AI) in healthcare: A review. ScienceOpen Preprints. 
[16] Sun, T.Q. and Medaglia, R., 2019. Mapping the challenges of Artificial Intelligence in the public sector:
Evidence from public healthcare. Government Information Quarterly, 36(2), 368-383. 